\title{
Multistratum Space-Time Codes for the Asynchronous Uplink of MIMO-CDMA Systems
}

\author{
Ronald Böhnke, Volker Kühn, and Karl-Dirk Kammeyer \\ Department of Communications Engineering \\ University of Bremen \\ Otto-Hahn-Allee, 28359 Bremen, Germany \\ Email: \{boehnke, kuehn, kammeyer\}@ant.uni-bremen.de
}

\begin{abstract}
We propose the use of multistratum space-time codes (MSSTC) for the asynchronous uplink of broadband MIMO-CDMA systems. This transmission scheme, originally proposed for single-user systems and flat fading channels, combines the benefits of both space-time block codes and multilayer transmission, i.e. it exploits transmit diversity and simultaneously enables high data rates with reasonable decoding complexity. Though the full potential of multistratum codes can only be achieved by using adaptive channel coding, we show by simulation results that they are a promising approach even in the uncoded case.
\end{abstract}

Index Terms-Multiuser MIMO systems, Space-Time Codes, CDMA, Uplink.

\section{INTRODUCTION}

In [1] it was shown, that channel capacity increases dramatically by using multiple transmit and receive antennas in rich scattering environments. Layered transmission strategies like D-BLAST [2] or V-BLAST [3] are a practical way to realize a considerable fraction of the capacity offered by such multipleinput multiple-output (MIMO) systems. The detection is typically based on successive interference cancellation (SIC). On the other hand, the transmission quality may be significantly improved by making use of spatial diversity. In order to gain diversity at the transmitter site, space-time coding has to be applied [4]. A special case are space-time block codes (STBC), which allow very simple decoding [5], [6].

These two concepts are combined in multistratum spacetime coding (MSSTC) [7]. Here, parallel data streams (called strata) are individually encoded using a space-time block code, respectively, and subsequently superimposed. Due to the STBC, each stratum contains full transmit diversity. In this paper, we analyze the applicability of multistratum space-time codes for the asynchronous uplink of MIMO-CDMA systems with frequency-selective channels. Furthermore, an efficient detection scheme for this scenario is devised.

In Section II the system model is introduced. V-BLAST and space-time block codes are reviewed in Section III and Section IV, respectively, before the multistratum codes are introduced in Section V. Section VI includes simulation results and concluding remarks can be found in Section VII.

This work was supported in part by the German ministry of education and research (BMBF) under grant $01 \mathrm{BU} 153$.

\section{System Model}

Throughout this paper, we use bold lower (upper) case letters for vectors (matrices) and an underline to indicate complex quantities. For an arbitrary complex $m \times n$ matrix $\underline{\mathbf{M}}$ we define the corresponding real $2 m \times n$ matrix $\mathbf{M}$, that alternately contains the real and imaginary part of each row.

We consider a MIMO-CDMA system, where $N_{U}$ users transmit over $N_{T}$ antennas to a common base station equipped with $N_{R}$ receive antennas. Each user is assigned a unique pseudo noise spreading code $\underline{c}_{u}[k]$ of length $N_{C}$, such that $\underline{c}_{u}[k]=0$ for $k<0$ or $k \geq N_{C}$. The channel between transmit antenna $t$ of user $u$ and receive antenna $r$ is characterized by the delay $\tau_{r, t, u}=\tau_{u}$ and the time-discrete impulse response in the equivalent baseband $\underline{h}_{r, t, u}[k]$ of order $N_{H}$. Without loss of generality we assume that $\tau_{u} \leq \tau_{u^{\prime}}$ for $u<u^{\prime}$ and $\tau_{1}=0$. As the receiver can only observe the linear convolution of channel and spreading code, we introduce the signature sequences

$$
\underline{s}_{r, t, u}[k]=\underline{h}_{r, t, u}[k] * \underline{c}_{u}\left[k-\tau_{u}\right],
$$

which are zero for $k<\tau_{u}$ or $k \geq N_{C}+N_{H}+\tau_{u}$. Note that the signatures may easily be replaced by their oversampled versions, but here we restrict to chip synchronous situations for simplicity.

Denoting the $i$-th transmit symbol (before spreading) of user $u$ at antenna $t$ by $\underline{x}_{t, u}[i]$, the receive signal at antenna $r$ is

$$
\underline{y}_{r}[k]=\sum_{i=0}^{\infty} \sum_{u=1}^{N_{U}} \sum_{t=1}^{N_{T}} \underline{s}_{r, t, u}\left[k-i N_{C}\right] \underline{x}_{t, u}[i]+\underline{n}_{r}[k],
$$

where $\underline{n}_{r}[k]$ represents complex white Gaussian noise of variance $\sigma_{n}^{2}$.

The system model (2) can be written in more compact form by making use of matrix notation. To this end, let us define the $N_{C} N_{R} \times N_{U} N_{T}$ signature matrix

$$
\underline{\mathbf{S}}[k]=\left(\begin{array}{llll}
\underline{\mathbf{S}}_{1}[k] & \underline{\mathbf{S}}_{2}[k] & \ldots & \underline{\mathbf{S}}_{N_{U}}[k]
\end{array}\right),
$$

where the $N_{R} \times N_{T}$ submatrix

$$
\underline{\mathbf{S}}_{u}[k]=\left(\begin{array}{ccc}
\underline{s}_{1,1, u}[k] & \cdots & \underline{s}_{1, N_{T}, u}[k] \\
\vdots & \ddots & \vdots \\
\underline{s}_{N_{R}, 1, u}[k] & \cdots & \underline{s}_{N_{R}, N_{T}, u}[k]
\end{array}\right)
$$


contains the contribution of user $u$ at time $k$. Similarly, we collect the transmit symbols, receive signals, and noise samples in the vectors

$$
\begin{aligned}
\underline{\mathbf{x}}[i] & =\left(\underline{\mathbf{x}}_{1}^{T}[i], \underline{\mathbf{x}}_{2}^{T}[i], \ldots, \underline{\mathbf{x}}_{N_{U}}^{T}[i]\right)^{T} \\
\text { with } \underline{\mathbf{x}}_{u}[i] & =\left(\underline{x}_{1, u}[i], \underline{x}_{2, u}[i], \ldots, \underline{x}_{N_{T}, u}[i]\right)^{T}, \\
\underline{\mathbf{y}}[k] & =\left(\underline{y}_{1}[k], \underline{y}_{2}[k], \ldots, \underline{y}_{N_{R}}[k]\right)^{T}, \\
\underline{\mathbf{n}}[k] & =\left(\underline{n}_{1}[k], \underline{n}_{2}[k], \ldots, \underline{n}_{N_{R}}[k]\right)^{T},
\end{aligned}
$$

respectively. With these definitions, the system model becomes

$$
\underline{\mathbf{y}}[k]=\sum_{i=0}^{\infty} \underline{\mathbf{S}}\left[k-i N_{C}\right] \underline{\mathbf{x}}[i]+\underline{\mathbf{n}}[k] .
$$

In general, a space-time codeword of one user consists of $N_{S T}$ consecutive transmit vectors and contains $K_{S T}$ information symbols

$$
\underline{\mathbf{d}}_{u}[m]=\left(\underline{d}_{u}\left[m K_{S T}\right], \ldots, \underline{d}_{u}\left[(m+1) K_{S T}-1\right]\right)^{T} .
$$

Usually, a codeword also includes conjugate complex information symbols. As complex conjugation is not a linear operation, it is necessary to split $\underline{\mathbf{d}}_{u}[\mathrm{~m}]$ up into real and imaginary part as described at the beginning of this section. With the resulting $2 K_{S T} \times 1$ vector $\mathbf{d}_{u}[m]$ and the $N_{S T}$ code defining generator matrices $\underline{\mathbf{G}}[i]$ of size $N_{T} \times 2 K_{S T}$ we can express the $i$-th symbol of the $m$-th space-time codeword by

$$
\underline{\mathbf{x}}_{u}\left[m N_{S T}+i\right]=\underline{\mathbf{G}}[i] \mathbf{d}_{u}[m] \quad, 0 \leq i<N_{S T} .
$$

Thus, the corresponding vector containing the transmit symbols of all users reads

$$
\underline{\mathbf{x}}\left[m N_{S T}+i\right]=\left(\mathbf{I}_{N_{U}} \otimes \underline{\mathbf{G}}[i]\right) \mathbf{d}[m] \quad, 0 \leq i<N_{S T},
$$

where $\otimes$ denotes the Kronecker product and $\mathbf{d}[m]$ contains the information symbols of all users.

Now, assuming that $\tau_{N_{U}}+N_{H} \leq N_{S T} N_{C}$, we can stack all nonzero signature matrices on top of each other in the $\left(N_{S T}+1\right) N_{C} N_{R} \times N_{U} N_{T}$ matrix

$$
\underline{\mathbf{S}}=\left(\begin{array}{llll}
\underline{\mathbf{S}}^{T}[0] & \underline{\mathbf{S}}^{T}[1] & \ldots & \left.\underline{\mathbf{S}}^{T}\left[\left(N_{S T}+1\right) N_{C}-1\right]\right)^{T} .
\end{array}\right.
$$

Then, after inserting (11) into (8), we find that the whole contribution of the information symbols $\underline{\mathbf{d}}[\mathrm{m}]$ to the receive signal (after space-time block coding, spreading, and linear distortion by the channel) is fully determined by the $2 N_{S T} N_{C} N_{R} \times 2 N_{U} K_{S T}$ system matrix

$$
\underline{\mathbf{A}}=\left(\begin{array}{cccc}
\underline{\mathbf{S}} & \mathbf{0} & \ldots & \mathbf{0} \\
\mathbf{0} & \underline{\mathbf{S}} & \ldots & \mathbf{0} \\
\vdots & \ddots & \vdots & \\
\mathbf{0} & \mathbf{0} & \ldots & \underline{\mathbf{S}}
\end{array}\right)\left(\begin{array}{c}
\mathbf{I}_{N_{U}} \otimes \underline{\mathbf{G}}[0] \\
\mathbf{I}_{N_{U}} \otimes \underline{\mathbf{G}}[1] \\
\vdots \\
\mathbf{I}_{N_{U}} \otimes \underline{\mathbf{G}}\left[N_{S T}-1\right]
\end{array}\right),
$$

where the zero submatrices of the block convolution matrix have dimension $N_{C} N_{R} \times N_{U} N_{T}$. For frequency-selective or asynchronous channels, every space-time codeword experiences interference from the previous and the next one.
Therefore, we split $\underline{\mathbf{A}}$ into its upper and lower halve $\underline{\mathbf{A}}_{u}$ and $\underline{\mathbf{A}}_{l}$, respectively, and introduce the matrices

$$
\underline{\mathbf{A}}_{p}=\left(\begin{array}{l}
\underline{\mathbf{A}}_{l} \\
\mathbf{0}
\end{array}\right) \quad \text { and } \quad \underline{\mathbf{A}}_{n}=\left(\begin{array}{c}
\mathbf{0} \\
\underline{\mathbf{A}}_{u}
\end{array}\right)
$$

having the same dimension as $\underline{\mathbf{A}}$, which characterize the interference from $\mathbf{d}[m-1]$ and $\mathbf{d}[m+1]$. With these definitions, the receive signal $\mathbf{y}[k]$ for $m N_{S T} N_{C} \leq k<(m+2) N_{S T} N_{C}$ can be merged in the vector

$$
\underline{\mathbf{y}}=\underline{\mathbf{A}}_{p} \mathbf{d}[m-1]+\underline{\mathbf{A}} \mathbf{d}[m]+\underline{\mathbf{A}}_{n} \mathbf{d}[m+1]+\underline{\mathbf{n}},
$$

where the vector $\underline{\mathbf{n}}$ includes the corresponding noise samples. Finally, as the symbols $\mathbf{d}[m]$ that need to be estimated at the receiver are real, we also partition $\underline{\mathbf{y}}, \underline{\mathbf{A}}_{p}, \underline{\mathbf{A}}, \underline{\mathbf{A}}_{n}$, and $\underline{\mathbf{n}}$ into their real and imaginary parts and arrive at the desired realvalued linear system model

$$
\mathbf{y}=\mathbf{A}_{p} \mathbf{d}[m-1]+\mathbf{A} \mathbf{d}[m]+\mathbf{A}_{n} \mathbf{d}[m+1]+\mathbf{n},
$$

which will be used throughout this paper. Note that with the normalization $\mathrm{E}\left\{\operatorname{tr}\left\{\mathbf{A}^{T} \mathbf{A}\right\}\right\}=2 N_{U} K_{S T}$, the average signal to noise ratio at the receiver for $M$-QAM is simply

$$
\frac{\sigma_{d}^{2}}{\sigma_{n}^{2}}=\frac{\sigma_{\underline{d}^{2}}}{\sigma_{\underline{n}^{2}}}=\log _{2}(M) \frac{E_{b}}{N_{0}},
$$

where $E_{b}$ is the mean energy per information bit and $N_{0}$ is the one-sided spectral power density of the noise.

\section{V-BLAST}

The aim of V-BLAST is to increase the data rate by using multiple antennas at both the transmitter and the receiver. For this purpose, independent data streams (called layers) are transmitted over the different antennas. Thus, it is possible to transmit $K_{S T} / N_{S T}=N_{T}$ symbols per channel use. The $N_{T} \times 2 N_{T}$ generator matrix reads

$$
\underline{\mathbf{G}}=\underline{\mathbf{G}}[0]=\mathbf{I}_{N_{T}} \otimes\left(\begin{array}{ll}
1 & j
\end{array}\right) \text {. }
$$
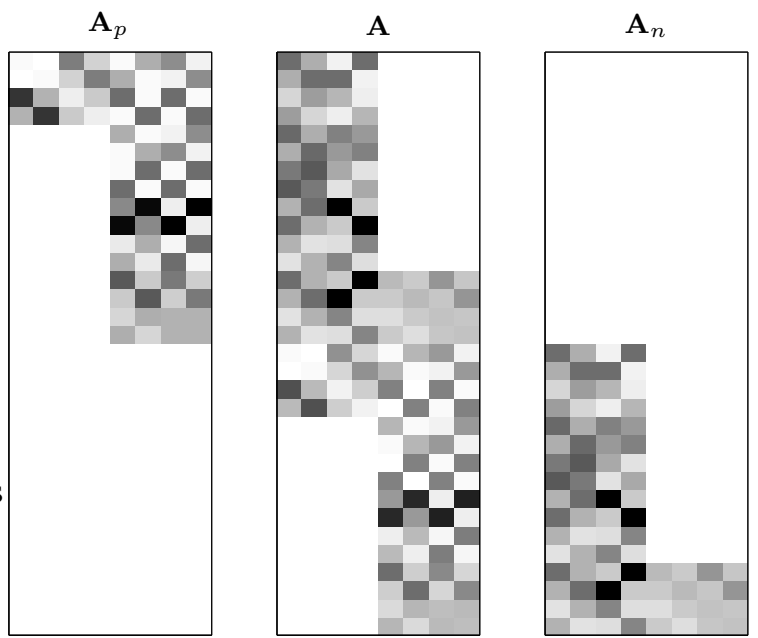

Fig. 1. V-BLAST system matrix for $N_{U}=2$ users with $N_{T}=2$ transmit antennas each, spreading factor $N_{C}=4$, channel order $N_{H}=1$, delay $\tau_{2}=3$, and $N_{R}=2$ receive antennas at the base station 
An example of the resulting real system matrix $\mathbf{A}$ (together with $\mathbf{A}_{p}$ and $\mathbf{A}_{n}$ ) is illustrated in Fig. $\mathbf{1}$ for an asynchronous two-user system. Here, dark entries correspond to large absolute values. The dimension of the system matrix is $4 N_{C} N_{R} \times 2 N_{U} N_{T}=32 \times 8$, where the first (last) two pairs of columns correspond to the antennas of user one (two), and each chip requires $2 N_{R}=4$ rows, so one complete signature sequence takes $2\left(N_{C}+N_{H}\right) N_{R}=20$ rows while the next symbol starts after $2 N_{C} N_{R}=16$ rows.

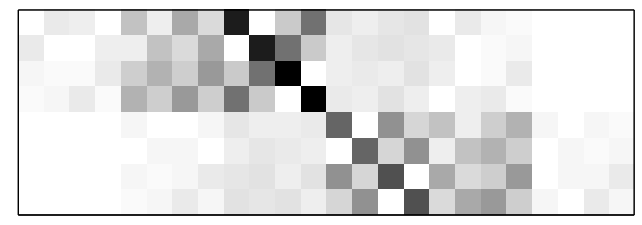

Fig. 2. Interference after matched filtering by $\mathbf{A}^{T}$

From Fig. 2 it can be observed that after simple matched filtering there remains strong co-channel interference from all other antennas as well as intersymbol interference from the previous and the next symbols. Therefore, more sophisticated detection techniques are required. The optimum maximum likelihood sequence estimation would be too complex for realtime applications, even with efficient implementations like the sphere decoder [8], that do not require an exhaustive search. For flat fading channels, a near-optimum detection algorithm based on lattice reduction was proposed in [9]. However, here we restrict to simple successive interference cancellation, which in many practical situation comes close to ML performance with a complexity comparable to linear detection.

Ignoring the noise for the moment and assuming that the receiver has already estimated $\mathbf{d}[m-1]$ correctly, the interference of the previous symbols can be subtracted from the receive signal. From (16) we get

$$
\left(\begin{array}{ll}
\mathbf{A} & \mathbf{A}_{n}
\end{array}\right)\left(\begin{array}{c}
\mathbf{d}[m] \\
\mathbf{d}[m+1]
\end{array}\right)=\mathbf{y}-\mathbf{A}_{p} \mathbf{d}[m-1] .
$$

The interference from yet undetected symbols needs to be suppressed by linear filtering. To this end, let us define the QL decomposition of

$$
\left(\begin{array}{ll}
\mathbf{A} & \mathbf{A}_{n}
\end{array}\right)=\mathbf{Q} \mathbf{L}=\left(\begin{array}{ll}
\mathbf{Q}_{1} & \mathbf{Q}_{2}
\end{array}\right)\left(\begin{array}{cc}
\mathbf{L}_{11} & \mathbf{0} \\
\mathbf{L}_{21} & \mathbf{L}_{22}
\end{array}\right),
$$

where the $4 N_{C} N_{R} \times 4 N_{U} N_{T}$ matrix $\mathbf{Q}$ has orthogonal columns, $\mathbf{L}$ is a monic lower triangular matrix ${ }^{1}$, and the introduced submatrices of $\mathbf{Q}$ and $\mathbf{L}$ are of identical dimensions, respectively. As by definition $\mathbf{Q}_{1}$ is orthogonal to $\mathbf{Q}_{2}$, from (19) and (20) it follows that

$$
\mathbf{L}_{11} \mathbf{d}[m]=\mathbf{Q}_{1}^{T} \mathbf{y}-\mathbf{Q}_{1}^{T} \mathbf{A}_{p} \mathbf{d}[m-1] .
$$

Now $\mathbf{d}[m]$ can be estimated from top to bottom, while subtracting all the interference from previously decided symbols.

\footnotetext{
${ }^{1} \mathrm{We}$ assume that the system is not overloaded, i.e. $N_{U} N_{T} \leq N_{C} N_{R}$.
}

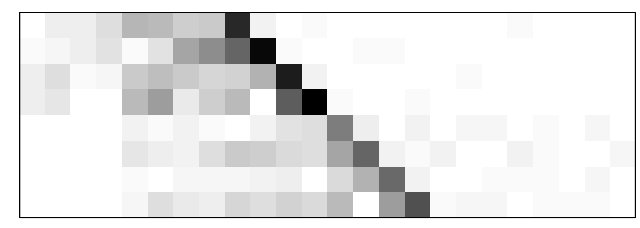

Fig. 3. Partial interference suppression by $\mathbf{Q}_{11}^{T}$

In [10] it was shown, that a linear minimum mean square error (MMSE) interference suppression (instead of the just described zero-forcing, which may lead to strong noise amplification) is achieved by considering the extended system matrix

$$
\left(\begin{array}{cc}
\mathbf{A} & \mathbf{A}_{n} \\
\sigma_{n} / \sigma_{d} \mathbf{I}
\end{array}\right)=\left(\begin{array}{ll}
\mathbf{Q}_{11} & \mathbf{Q}_{12} \\
\mathbf{Q}_{21} & \mathbf{Q}_{22}
\end{array}\right)\left(\begin{array}{cc}
\mathbf{L}_{11} & \mathbf{0} \\
\mathbf{L}_{21} & \mathbf{L}_{22}
\end{array}\right)
$$

and replacing $\mathbf{Q}_{1}$ in (21) by the matrix $\mathbf{Q}_{11}$ of the same size. Furthermore, a very efficient sorting algorithm to determine the optimum order of detection was proposed. The result of the partial interference suppression is shown in Fig. 3.

\section{Space-Time Block Codes}

Space-time block codes are a means to exploit spatial diversity in rich scattering environments in order to improve the transmission quality when no or only little temporal or frequency diversity is available. This is typically the case for indoor scenarios. The most simple example is the well-known Alamouti scheme [5] for $N_{T}=2$ transmit antennas with the unitary generator matrix

$$
\underline{\mathbf{G}}=(\underline{\mathbf{G}}[0])=\left(\begin{array}{rrrr}
1 & j & 0 & 0 \\
0 & 0 & 1 & j \\
\hline 0 & 0 & -1 & j \\
1 & -j & 0 & 0
\end{array}\right) .
$$

Here, only $K_{S T} / N_{S T}=1$ symbol is transmitted per channel use as opposed to V-BLAST, which on the other hand offers no transmit diversity. Also, due to $N_{S T}=2$, the number of rows of the resulting system matrix $\mathbf{A}$ is doubled compared to V-BLAST while the number of columns remains the same.

For a flat fading channel and synchronous users employing orthogonal spreading it can be easily verified that $\mathbf{A}$ has orthogonal columns. Hence, ML performance is achieved by simple matched filtering followed by a symbol-wise decision. However, for the example from the previous section these conditions are not fulfilled. Thus, the matched filter output contains residual interference, which is visible in Fig. 4, but obviously the signal to interference ratio (SIR) is much higher than for V-BLAST in Fig. 2.

Note that for $N_{T}>2$ there exists no linear orthogonal space-time block code with rate one. For this case, orthogonal codes with reduced rate [6], quasi-orthogonal codes [11], and nonlinear orthogonal codes [12] were proposed. Furthermore, for frequency-selective channels, special spacefrequency codes have been designed [13]. However, these codes need to be optimized for the actual power delay profile of the channel. Therefore, we restrict to the more practical 


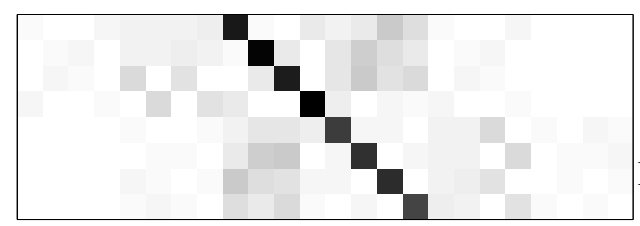

Fig. 4. Interference after matched filtering for space-time block coding and system parameters given in Fig. 1

approach to collect frequency diversity separately by the spreading code.

\section{Multistratum Space-Time Codes}

Multistratum space-time codes were first proposed in [7] for single-user systems and flat fading channels. They are a special case of the more general linear dispersion space-time codes [14] and combine the benefits of both space-time block codes and V-BLAST, i.e. they exploit transmit diversity and at the same time allow for high data rates while maintaining low decoding complexity by successive detection.

Similar to V-BLAST, the information symbols are demultiplexed into $N_{S}$ parallel data streams

$$
\mathbf{d}_{u}[m]=\left(\mathbf{d}_{1, u}^{T}, \mathbf{d}_{2, u}^{T}, \ldots, \mathbf{d}_{N_{S}, u}^{T}\right)^{T},
$$

which are referred to as strata. Instead of directly transmitting these symbols, all strata are first encoded with the same space-time block code and afterwards the resulting transmit vectors are superimposed using an orthogonal transform, e.g. the discrete fourier transform (DFT). Another interpretation of this encoding procedure is that every stratum employs its own characteristic space-time block code defined by

$$
\underline{\mathbf{G}}_{l}[i]=e^{-j 2 \pi l i / N_{S T}} \underline{\mathbf{G}}^{\prime}[i] \quad, 1 \leq l \leq N_{S},
$$

where $\underline{\mathbf{G}}^{\prime}[i]$ is the generator matrix of the common code and the DFT was used. Then, the whole generator matrix of the multistratum space-time code becomes

$$
\underline{\mathbf{G}}=\left(\begin{array}{ccc}
\underline{\mathbf{G}}_{0}[0] & \cdots & \underline{\mathbf{G}}_{N_{S}}[0] \\
\vdots & \ddots & \vdots \\
\underline{\mathbf{G}}_{0}\left[N_{S T}-1\right] & \cdots & \underline{\mathbf{G}}_{N_{S}}\left[N_{S T}-1\right] .
\end{array}\right)
$$

For the Alamouti scheme from (23) and $N_{S}=2$ strata this leads to

$$
\underline{\mathbf{G}}=\left(\begin{array}{rrrr|rrrr}
1 & j & 0 & 0 & 1 & j & 0 & 0 \\
0 & 0 & 1 & j & 0 & 0 & 1 & j \\
\hline 0 & 0 & -1 & j & 0 & 0 & 1 & -j \\
1 & -j & 0 & 0 & -1 & j & 0 & 0
\end{array}\right) .
$$

Compared to V-BLAST, the number of rows and columns in the system matrix has doubled. This does not necessarily mean a large increase of complexity, because for multistratum space-time codes A may be highly structured. Fig. 5 shows a simple example for a single user with $\mathbf{G}$ from (27) in a flat fading environment. While symbols of the same stratum do not interact due to the orthogonality of the space-time block code, there is strong interference among the strata. The $8 \times 8$ matrix a) $\mathbf{A}^{T} \mathbf{A}$

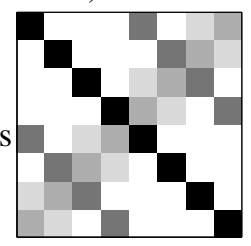

b) $\mathbf{L}$

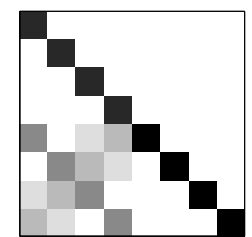

Fig. 5. Structure of the system matrix for a single user in flat fading

L used for the SIC is depicted in Fig. 5b. Only five different parameters (the upper left element and the last row) need to be calculated; for V-BLAST, $\mathbf{L}$ would only have size $4 \times 4$, but still contain four different values. However, in presence of intersymbol or multiuser interference this structure gets lost. This is demonstrated in Fig. 6, where the parameters from the example in Section III where used.

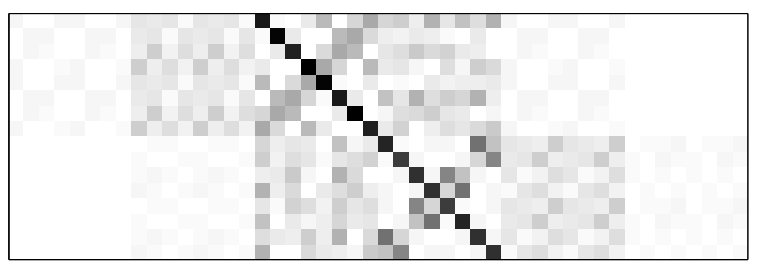

Fig. 6. Interference after matched filtering for multistratum space-time coding and system parameters given in Fig. 1

From Fig. 5a it is obvious that all information symbols have the same SIR. Therefore, in contrast to the layers in V-BLAST, the strata can be decoded in arbitrary order, so no sorting of the strata is required. This allows for adaptive coding of the strata according to the remaining interference, i.e. the stratum detected first may employ a strong channel code while the code rate can be gradually increased for the remaining strata due to the SIC. This is not possible for V-BLAST. Note, however, that for an uncoded transmission it is advantageous to decide only one symbol of each stratum at a time and then move on to the next symbol instead of detecting the whole stratum, because interference cancellation only improves the performance of all other strata, but not the current one.

Another important advantage of multistratum codes is, that they allow for a flexible trade-off between data rate and error performance by simply switching off strata, which does not lead to transmission breaks. Note that for $N_{S}=1$ the multistratum approach degenerates to ordinary space-time block coding.

\section{Simulation Results}

The resulting bit error rates (BER) for uncoded QPSK transmission of a single user with $N_{T}=2$ transmit antennas and an uncorrelated flat Rayleigh fading channel are shown in Fig. 7. For detection, the receiver applies SIC with MMSE interference suppression and optimum sorting as described in Section III. Surprisingly, for $N_{R} \leq 4$, multistratum spacetime codes (MSSTC) perform worse than V-BLAST. This is 


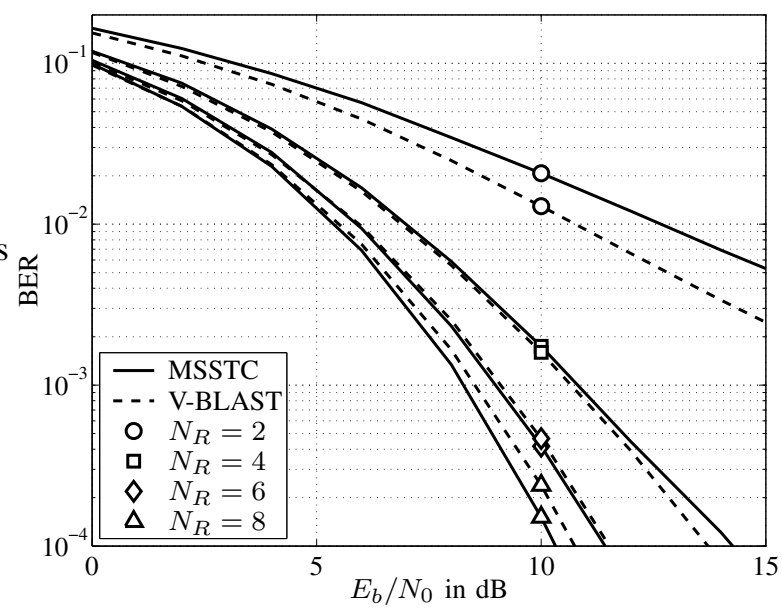

Fig. 7. BER for a single user with $N_{T}=2$ antennas in flat fading

because the first stratum experiences strong interference from the second one. As mentioned in the previous section, this may be accounted for by adaptive coding. However, with increasing number of receive antennas, multistratum codes are superior even in the uncoded case, because then the first stratum can be detected sufficiently well and the diversity advantage of space-time coding becomes predominant. Note that in many practical scenarios the number of transmit antennas will be rather small due to space and cost limitations of the mobile unit, while a fixed base station may utilize a large number of receive antennas.

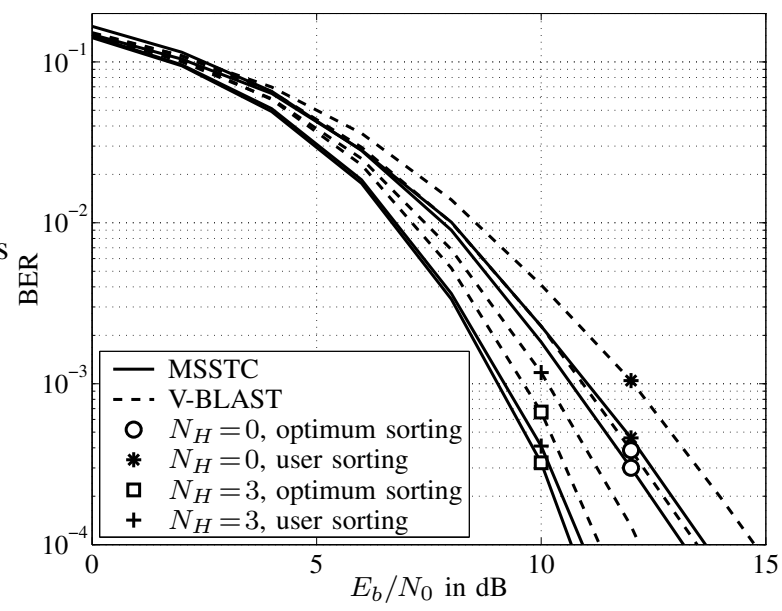

Fig. 8. BER for $N_{U}=8$ asynchronous users with $N_{T}=2$ transmit antennas each, spreading factor $N_{C}=4$, and $N_{R}=6$ receive antennas

In Fig. 8, we analyze the BER performance of a MIMOCDMA system with eight asynchronous users. While in the previous example both transmission schemes were nearly identical for $N_{R}=6$ receive antennas, multistratum spacetime coding now clearly outperforms V-BLAST, especially for the frequency-selective channel. The reason for this behavior is, that the high interference between strata of one user, that caused the performance degradation, is now dominated by the multiuser (and maybe intersymbol) interference, which is comparable for both multistratum codes and V-BLAST. Finally, we find that the receiver complexity can be significantly reduced by only optimizing the detection order over the $N_{U}=8$ users instead of all $2 N_{U} N_{S} K_{S T}=64$ real symbols. For multistratum codes this causes only a small performance loss, while for V-BLAST using the correct ordering is essential.

\section{CONCLUSIONS}

We demonstrated that multistratum space-time codes can significantly improve the performance of asynchronous CDMA uplink transmission. Using appropriate adaptive channel coding, which is not possible for V-BLAST, the gain will certainly be even larger. For system parameters of practical interest, the devised frequency-selective SIC detection algorithm based on a QL decomposition of the system matrix is suitable for real-time applications like e.g. video conferencing. Note that for $N_{T}>2$, linear orthogonal space-time codes always involve a rate loss. Hence, the application of quasi-orthogonal codes would be an interesting topic for future work.

\section{REFERENCES}

[1] E. Telatar, "Capacity of Multi-antenna Gaussian Channels," European Transactions on Telecommunications, vol. 10, no. 6, pp. 585-595, November-December 2000.

[2] G. J. Foschini, "Layered Space-Time Architecture for Wireless Communication in a Fading Environment when Using Multiple Antennas," Bell Labs Technical Journal, vol. 1, no. 2, pp. 41-59, Autumn 1996.

[3] P. W. Wolniansky, G. J. Foschini, G. D. Golden, and R. A. Valenzuela, "V-BLAST: An Architecture for Realizing Very High Data Rates Over the Rich-Scattering Wireless Channel," in Proc. ISSE, Pisa, Italy, September 1998.

[4] V. Tarokh, N. Seshadri, and A. R. Calderbank, "Space-Time Codes for High Data Rate Wireless Communication: Performance Criterion and Code Construction," IEEE Transactions on Information Theory, vol. 44, no. 2, pp. 744-765, March 1998.

[5] S. M. Alamouti, "A Simple Transmit Diversity Technique for Wireless Communications," IEEE Journal on Selected Areas in Commununications, vol. 16, no. 8, pp. 1451-1458, October 1998.

[6] V. Tarokh, H. Jafarkhani, and A. R. Calderbank, "Space-Time Block Codes from Orthogonal Designs," IEEE Transactions on Information Theory, vol. 45, no. 5, pp. 1456-1467, July 1999.

[7] U. Wachsmann, J. Thielecke, and H. Schotten, "Exploiting the DataRate Potential of MIMO Channels: Multi-Stratum Space-Time Coding," in Proc. IEEE VTC Spring 2001, Rhodes, Greece, May 6-9 2001.

[8] E. Agrell, T. Eriksson, A. Vardy, and K. Zeger, "Closest Point Search in Lattices," IEEE Transactions on Information Theory, vol. 48, no. 8, pp. 2201-2214, August 2002.

[9] D. Wübben, R. Böhnke, V. Kühn, and K. D. Kammeyer, "Near Maximum-Likelihood Detection of MIMO Systems using MMSE-Based Lattice Reduction,” accepted at ICC 2004, Paris, France, June 20-24 2004.

[10] R. Böhnke, D. Wübben, V. Kühn, and K. D. Kammeyer, "Reduced Complexity MMSE Detection for BLAST Architectures," in Proc. IEEE Global Communications Conference (Globecom'03), San Francisco, California, USA, December 2003.

[11] H. Jafarkhani, "A Quasi-Orthogonal Space-Time Block Code," IEEE Transactions on Communications, vol. 49, no. 1, pp. 1-4, January 2001.

[12] L. He and H. Ge, "A New Full-Rate Full-Diversity Orthogonal SpaceTime Block Coding Scheme," IEEE Communications Letters, vol. 7, no. 12, pp. 590-592, December 2003.

[13] W. Su, Z. Safar, and K. Liu, "Full-Rate Full-Diversity Space-Frequency Codes With Optimum Coding Advantage," IEEE Transactions on Information Theory, September 2003, submitted for publication.

[14] B. Hassibi and B. Hochwald, "High-Rate Codes that are Linear in Space and Time," IEEE Transactions on Information Theory, vol. 48, no. 7, pp. 1804-1824, July 2002. 